\title{
Second Heart Sound Onset to Identify T-Wave Offset
}

\author{
Agnese Sbrollini ${ }^{1}$, Marta Beghella Bartoli ${ }^{1}$, Angela Agostinelli ${ }^{1}$, Micaela Morettini ${ }^{1}$, \\ Francesco Di Nardo ${ }^{1}$, Sandro Fioretti ${ }^{1}$, Laura Burattini ${ }^{*}, 1$ \\ ${ }^{1}$ Università Politecnica delle Marche, Ancona, Italy
}

\begin{abstract}
Phonocardiography (PCG) second heart sound represents aortic-pulmonary valves closure and beginning of isovolumetric relaxation of the ventricles. Electrocardiography (ECG) $T$ wave represents electrical repolarization of the ventricles. Ventricular electrical repolarization is known to drive ventricular mechanical relaxation. Thus, the aim of the present study was to investigate whether, in normal conditions, second heart sound onset (S2on) matches in time T-wave offset (Toff) so that S2on may be used to identify Toff. To this aim, 99 couples of simultaneously recorded short (around $30 \mathrm{~s}$ $P C G$ and ECG) signals relative to normal subjects (selected from PhysioNet/CinC Challenge 2016: Training Set A) were analyzed. S2on was identified by application of our newly developed threshold-based algorithm to the median beat of the PCG envelope. Instead, Toff was identified by application of the Laguna and Thakor algorithm to the median ECG beat. Median time-distance $(\delta t)$ between S2on and Toff was $5 \mathrm{~ms}(P=0.007)$. Thus, in normal conditions, S2on and Toff differ on average of 5 ms, whose meaning remain to be defined. Still, $5 \mathrm{~ms}$ is included in the Toff identification variability (of the order of tens ms) due to different Toff identification methods and electrocardiographic leads. Consequently, in normal condition, S2on may be used to estimate Toff.
\end{abstract}

\section{Introduction}

Phonocardiogram (PCG) and electrocardiogram (ECG) are two of the most popular non-invasive techniques for cardiac monitoring [1,2]. PCG is the recording of heartsounds and provides information about the cardiac mechanical behavior. Specifically, in normal conditions, PCG is composed by the repetition of two heart sounds. The first heart sound (S1) represents the closure of atrioventricular (mitral and tricuspid) valves and the beginning of ventricular contraction (systole). The second heart sound (S2) represents the closure of semilunar (aortic and pulmonary) valves and the beginning of isovolumetric relaxation of the ventricles (diastole).

ECG is the recording of the cardiac electrical activity and provides information about the conduction system. Specifically, in normal condition, ECG is composed by the repetition of several waves: P wave, QRS complex and $\mathrm{T}$ wave. $\mathrm{P}$ wave and $\mathrm{QRS}$ complex represent atrial and ventricular depolarizations respectively, while $\mathrm{T}$ wave represents repolarization of ventricles (atrial repolarization is covered by the high amplitude of QRS complex).

T-wave offset (Toff) identification is one of the most important challenge in ECG analysis: Toff is fundamental to compute the major features for diagnosis, but suffers from lack of reliability due to the presence of noise [3]. Thus, indirect measures of Toff are desirable.

By observing simultaneous PCG and ECG recordings, it can be noted that Toff and S2 onset (S2on) seem to have the same time location in cardiac cycle (Figure 1). Previous studies [4-8] used this property for evaluating the QT/QS2 index, defined as the ratio between QT interval (time distances between beginning of QRS complex and end of T wave) and QS2 interval (time distances between beginning of QRS complex and first major vibration of aortic component of S2 [9], respectively). QT/QS2 index was found to be a distinctive feature between normal and pathological signals, with $\mathrm{QT} / \mathrm{QS} 2=1$ in normal conditions [10]. This result suggests that S2on may be used as an indirect measure of Toff. To further asses this issue, the present study aimed to investigate whether, in normal conditions, S2on matches Toff in time.

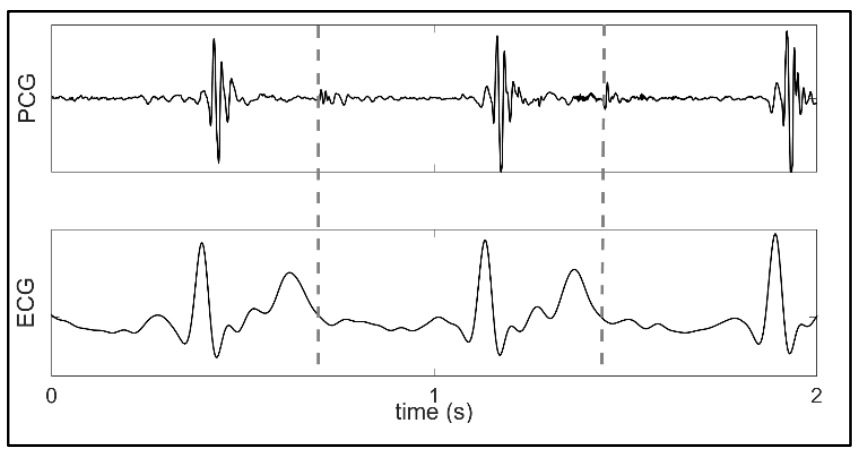

Figure 1. PCG and ECG simultaneously recorded. Dotted lines indicate the S2on and Toff locations. 


\section{Material and Methods}

\subsection{Data}

In this study, 99 couples of simultaneously recorded short (around $30 \mathrm{~s}$ ) PCG and ECG signals from normal subjects, where elaborated for S2on and Toff identification, respectively. All recordings belong to the training set A of PhysioNet/CinC Challenge 2016 database $[11,12]$ and were acquired using a Welch Allyn Meditron electronic stethoscope (frequency response: 20 $\mathrm{Hz} \div 20 \mathrm{kHz}$; initial sampling rate $44100 \mathrm{~Hz}$ successively reduced to $2000 \mathrm{~Hz}$ for PCGs and $1000 \mathrm{~Hz}$ for ECGs).

\subsection{Second Heart Sound onset and $T$ wave offset Extraction}

The block diagram of the procedure for S2on and Toff extraction is represented in Figure 2. The procedure included three steps: pre-processing, median beats computation and features extraction.

\subsubsection{Pre-Processing}

PCG and ECG were independently pre-processed. PCG was filtered with a low-pass filter (bidirectional $3^{\text {rd }}$ order Butterworth filter; cut-off frequency: $\mathrm{Hz}$ ) to obtain its envelope, ePCG. Instead, ECG was filtered with a band-pass filter (bidirectional $3^{\text {rd }}$-order Butterworth filter; cut-off frequencies: $0.5-45 \mathrm{~Hz}$ ) before R-peak detection by application of the Pan-Tompkins algorithm [13].

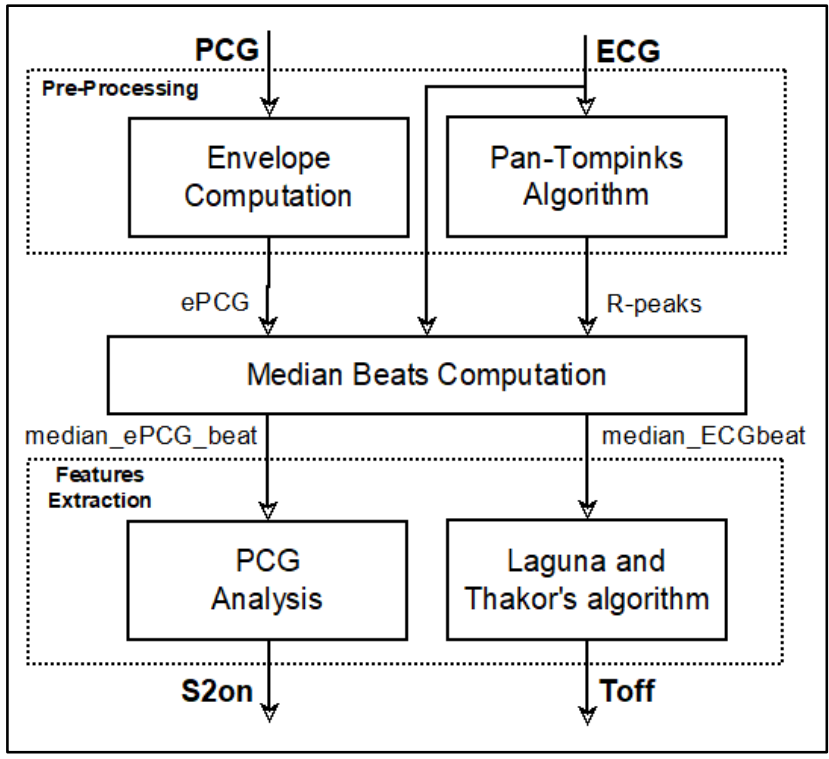

Figure 2. Block diagram of the procedure for S2on and Toff extraction.

\subsubsection{Median Beats Computation}

Being ePCG and ECG two simultaneous cardiac signals, they included the same number $\mathrm{N}$ of synchronized cardiac beats and were characterized by the same heart rate (given by the reciprocal of mean RR interval, mRR). Therefore, ePCG and ECG were both segmented into $\mathrm{N}$ beats using R-peaks positions as reference points. A segmented beat was included between $150 \mathrm{~ms}$ before the R-peak position and $\mathrm{mRR}-150 \mathrm{~ms}$ after the R-peak position. All segmented beats in ePCG were used to compute median_ePCG_beat; analogously, all segmented beats in ECG were used to compute the median_ECG_beat. For the properties of median computation, both median_ePCG_beat and median_ECG_beat were affected by a reduced amount of noise and remained aligned.

\subsubsection{Features Extraction}

Features extraction consisted in the extraction of S2on from median_ePCG_beat and of Toff from median_ECG_beat.

S2on was identified by application of our thresholdbased algorithm. Specifically, a threshold was computed as:

$$
\text { threshold }=2 \cdot \sigma_{\mathrm{ePCG}}
$$

where $\sigma_{\mathrm{ePCG}}$ is ePCG standard deviation. Theoretically, median_ePCG_beat crossed the threshold in four points: S1 onset, S1 offset, S2 onset and S2 offset. Therefore, the third point was the one of interest. To avoid misclassification, the time distance between $\mathrm{S} 1$ offset and S2 onset had to satisfy the following conditions:

$$
\text { S2 onset }- \text { S1 offset }\left\{\begin{array}{l}
>60 \mathrm{~ms} \\
\leq 450 \mathrm{~ms}
\end{array}\right.
$$

according to their physiological meaning.

Toff was identified by application of the Laguna and Thakor algorithm to median_ECG_beat. Details may be found in [14].

S2on (ms) was quantified as time interval from median_ePCG_beat beginning to S2 onset. Analogously, Toff was quantified as time interval from median_ECG_beat beginning to T offset.

\subsection{Statistics}

Misalignment between S2on and Toff was quantified by the time-distance between S2on and Toff, measured as:

$$
\delta \mathrm{t}=\mathrm{S} 2 \mathrm{on}-\mathrm{Toff}
$$




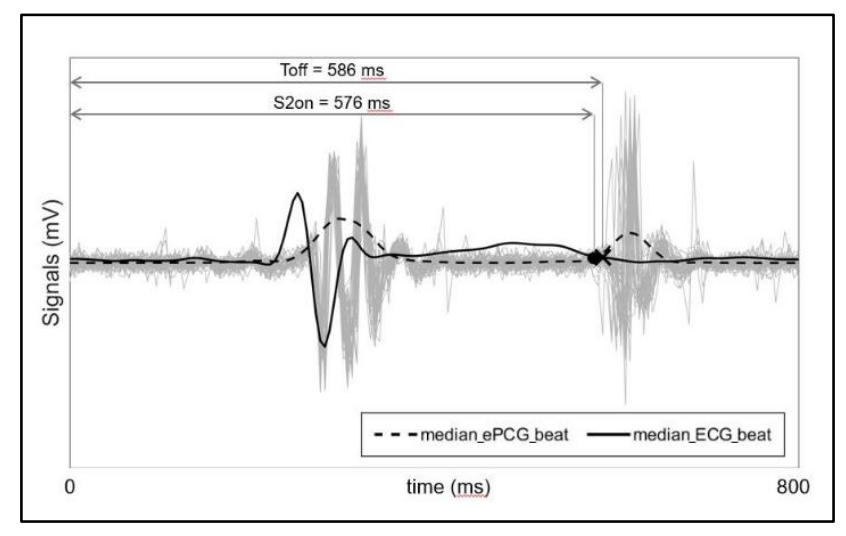

Figure 3. S2on $(\bullet)$ and Toff $(\times)$ identification from median_ePCG_beat, represented with a dotted line, and median_ECG_beat, represented with a solid line, respectively. Eventually, gray line represents the original PCG used to compute ePCG was computed

Association between features was evaluated using the Pearson correlation coefficient $(\rho)$ and the regression line. Non-normal parameter distributions were described in terms of $50^{\text {th }}\left[25^{\text {th }} ; 75^{\text {th }}\right]$ percentiles and comparted using the Wilcoxon Rank-Sum test for equal medians. Statistical significance was set at 0.05 in all cases.

\section{Results}

A representative example of S2on extraction from median_ePCG_beat and Toff extraction from median_ECG_beat is represented in Figure 3. In this case, although S2on (576 ms) visually almost matched Toff (586 ms), $\delta$ t is quite high (10 ms).

Overall, S2on and Toff distributions were characterized by not statistically different median values (S2on: 580 [560; 605] ms; Toff: 575 [555; 590] ms; $\mathrm{P}=0.086)$. Still, median value of $\delta \mathrm{t}$ distribution was statistically greater than zero $(5[-10 ; 25] \mathrm{ms} ; \mathrm{P}=0.007)$. In addition, S2on and Toff were associated by a correlation of $0.61\left(\mathrm{P}<10^{-12}\right)$ and regression line was S2on=0.6 Toff +200 (Figure 4$)$.

\section{Discussion}

The aim of the present study was to investigate whether S2on could represent a reliable indirect measurement of Toff. Indeed, S2on and Toff are different representation of the same physiological phenomenon. By considering that S2on indicates the time instant in which semilunar valves close and that Toff indicate the time instant in which ventricular repolarization ends, it follows that they are two measurements of heart repolarization ending. In order to compare S2on and Toff

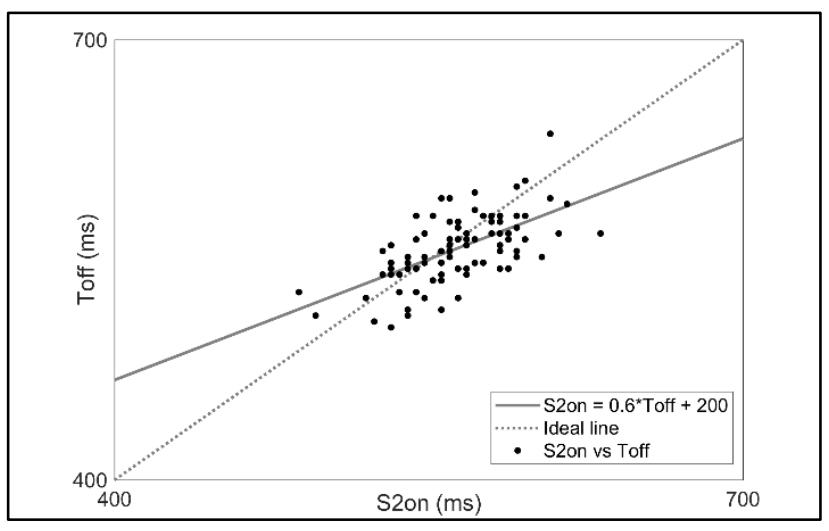

Figure 4. Regression line (solid line) between S2on and Toff. Ideal line (dotted line, corresponding to S2on=Toff) is depicted as reference.

measurements, 99 couples of simultaneously recorded PCG and ECG signals were analyzed. To avoid identification errors due to the presence of noise, S2on and Toff were identified on PCG and ECG median beats that. Indeed, for the median properties, median beats were affected by a significantly reduced level of noise with respect to their generating signals.

S2on was computed with our newly developed threshold-based technique. This new method was inspired by the De Luca's algorithm for identification of surface muscle activation from electromyographic signals [15]. Indeed, PCG is morphologically (but not physiologically) similar to surface electromyography: both signals could be modeled as stochastic processes oscillating around zero (mean value equal to zero). The procedure used by De Luca to find the onset and the offset of muscles activation was adapted here to identify the onset and the offset of heart sounds waveforms.

To asses S2on reliability in estimating ECG T-wave offset, S2on was compared to Toff from simultaneous signals. Among the several techniques available in literature for Toff identification, we chose Laguna and Thakor's algorithm [14], since very popular and particularly reliable.

According to our results, S2on and Toff distributions were not statistically different and were characterized by similar trends, as qualitatively demonstrated in Figure 4. Despite of that, correlation between S2on and Toff values was medium $\left(0.61 ; \mathrm{P}<10^{-12}\right)$, regression-line correlation coefficient and intercept (0.6 and $200 \mathrm{~ms}$, respectively) were different from those of the ideal-line $(1$ and $0 \mathrm{~ms}$, respectively), and $\delta$ t distribution was found to have a median value equal to $5 \mathrm{~ms}$. These results suggest that some differences in the S2on and Toff measures occur. Currently, the meaning of the time-distance between S2on and Toff remains unknown, even though two causes are conceivable and will be investigated by future studies. 
The first cause of the difference could have a physiological origin. Specifically, this difference could be due to a physiological delay occurring between cardiac electrical and mechanical activities. If this is the case, our results suggest that, since S2on is on average $5 \mathrm{~ms}$ longer than Toff, then semilunar valves close, on average, few milliseconds after the ending of ventricular electrical repolarization. The second cause of the difference between S2on and Toff could relate to noise and algorithms identification errors. Specifically, analyzed PCG and ECG may be affected by different levels of noise so that S2on and Toff identification accuracy could be different. Moreover, no studies have yet demonstrated that threshold value defined in Eq. 3 is optimal for heartsound tones identification from PCG. Future studies will investigate the real nature of $\delta$ t delay, considering the physiological mechanism beyond the heart behavior and optimizing the threshold value of the S2on identification algorithm.

Despite of the median $5 \mathrm{~ms}$ delay of S2on with respect to Toff, S2on can still be used as an indirect measure of Toff, even without correcting it (that is without systematically subtracting $5 \mathrm{~ms}$ from its measure). Indeed, $5 \mathrm{~ms}$ is included in the accepted Toff identification variability (of the order of tens of ms [3]) usually observed when measuring Toff in different electrocardiographic leads, with different methods, and with different levels of noise. Thus, an average error of 5 $\mathrm{ms}$ is typically accepted when measuring Toff.

The clinical and practical utility of PCG S2on to have a measure of ECG Toff will be investigated in future studies that will use S2on to compute fundamental clinical features (such as the QT interval) to discriminate normal and pathological conditions.

\section{Conclusion}

In normal conditions, S2on may be used to indirectly estimate Toff, since the average delay occurring between them is small $(5 \mathrm{~ms})$ and within the range of acceptability of Toff identification variability.

\section{References}

[1] Schölzel C, Dominik A. Can electrocardiogram classification be applied to phonocardiogram data? - An analysis using recurrent neural networks. Computing in Cardiology 2016;43:581-4.

[2] Jain PK, Tiwari AK. Heart monitoring systems-A review. Compututers in Biology and Medicine 2014;54:1-13.

[3] Giuliani C, Agostinelli A, Di Nardo F, Fioretti S, Burattini L. Automatic Identification of the Repolarization Endpoint by Computing the Dominant T-wave on a Reduced Number of Leads. The Open Biomedical Engineering Journal 2016;10:43-50.

[4] Zdrojewski TR, Purzycki Z, Rynkiewicz A, Kubasik A,Wyrzykowski B, Krupa-Wojciechowska B. QT/QS2 ratio in mitral valve prolapse syndrome, hyperthyroidism and borderline hypertension: Possible indication of dysautonomia. Am J Noninvas Card 1993;7:19-22.

[5] Pietrzyk E, Sadowski Z, Szwed H, Kraska A. Prognostic value of QT-QS2 index in the assessment of sudden coronary death. Kardiologia Polska 1990;33:240-9.

[6] Assmann I, Kassel P, Kretzschmar E, Porstmann I. Behaviour of the electrical systole (QT) and the ratio of electromechanical systole (QS2)/QT in healthy persons and in patients with cardiac diseases at rest, under drug influence, and heart rate increasing. XVth International Congress on Electrocardiology 1988;57-60.

[7] Boudoulas H, Bush CA, Schall SF, Leier CV, Lewis RP. Prolonged electrical systole and QT > QS2 secondary to coronary artery disease. American Journal of Cardiology 1985;55:915-9.

[8] Boudoulas H, Sohn YH, O'neill W, Brown R, Weissler AM. The QT > QS2 syndrome: A new mortality risk indicator in coronary artery disease. American Journal of Cardiology 1982;50:1229-35.

[9] Ghai CL. A textbook of pratical physiology. New Delhi: Jaypee Brothers Medical Publishers (P) Ltd, 2013 ( $8^{\text {th }}$ edition).

[10] Wodniecki J, Foremny J, Bacior B, Braksator W, Kopeć P, Kreis W, Kubik L, Nessler J, Ostrowska B, PiktoPietkiewicz W. Ventricular arrhythmias and QT/QS2 index in patients with ischemic heart disease. Kardiologia Polska 1990;33:303-7.

[11] Liu C, Springer D, Li Q, Moody B, Juan RA, Chorro FJ, Castells F, Roig JM, Silva I, Johnson AE, Syed Z, Schmidt SE, Papadaniil CD, Hadjileontiadis L, Naseri $H$, Moukadem A, Dieterlen A, Brandt C, Tang H, Samieinasab M, Samieinasab MR, Sameni R, Mark RG, Clifford GD. An open access database for the evaluation of heart sound algorithms. Physiological Measurement 2016;37:2181-213.

[12] Goldberger AL, Amaral LA, Glass L, Hausdorff JM, Ivanov PC, Mark RG, Mietus JE, Moody GB, Peng CK, Stanley HE. PhysioBank, PhysioToolkit, and PhysioNet: components of a new research resource for complex physiologic signals. Circulation 2000;101:e215-20.

[13] Pan J, Tompkins WJ. A real-time QRS detection algorithm. IEEE Transactions on Biomedical Engineering 1985;32:230-6

[14] Laguna P, Thakor NV, Caminal P, Jané R, Yoon HR, Bayés de Luna A, Marti V, Guindo J. New algorithm for QT interval analysis in 24-hour Holter ECG: performance and applications. Medical \& Biological Engineering \& Computing. 1990;28 67-73.

[15] De Luca CI. The Use of Surface Electromyography in Biomechanics. Journal of Applied Biomechanics 1997;13:135-163.

Address for correspondence.

Laura Burattini.

Department of Information Engineering,

Università Politecnica delle Marche,

via Brecce Bianche 12,

60131, Ancona, Italy.

E-mail address. 1.burattini@univpm.it. 